\title{
Que peuvent apprendre les pays de la réponse de Hong Kong à la pandémie de COVID-19?
}

\author{
Samuel Y.S. Wong MD MPH, Kin On Kwok PhD, Francis K.L. Chan MD \\ - Citation : CMAJ 2020 May 11;192:E511-5. doi: 10.1503/cmaj. 200563-f; diffusion hâtive le 24 avril 2020 \\ Voir la version anglaise de l'article ici : www.cmaj.ca/lookup/doi/10.1503/cmaj.200563
}

H

ong Kong, une région administrative spéciale de la République populaire de Chine, se situe au sud du pays et partage une frontière avec la Chine continentale. Comptant plus de 7,45 millions d'habitants, Hong Kong est un important carrefour de déplacements dans la région : on y trouve le huitième aéroport le plus achalandé au monde, et plus de 70 millions de voyageurs y transitent chaque année. Hong Kong a également l'une des populations les plus denses de la planète, soit 17311 personnes par mille carré (6659 personnes par kilomètre carré). Étant donné la densité de sa population et sa proximité avec la Chine continentale, Hong Kong était donc particulièrement vulnérable à la pandémie de maladie à coronavirus 2019 (COVID-19).

Le premier cas de COVID-19 à Hong Kong a été signalé le 22 janvier 2020. De janvier à mars, 96709 tests de dépistage ont été effectués, et en date du 14 avril, 1010 personnes avaient été déclarées infectées par le coronavirus du syndrome respiratoire aigu sévère 2 (SRAS-CoV-2), et 4 étaient décédées de la COVID-191. Malgré sa situation particulière, Hong Kong a relativement bien contenu la propagation de la maladie (figure 1 ; annexe 1 , accessible [en anglais] au www.cmaj.ca/lookup/suppl/doi:10.1503/ cmaj.200563/-/DC1). Dans cet article, nous analysons les raisons pouvant expliquer pourquoi Hong Kong a obtenu de bons résultats et a bien réagi à la menace posée par le SRAS-CoV-2, afin que d'autres pays puissent tirer profit de son expérience.

\section{Qu'a fait Hong Kong pour maîtriser la COVID-19?}

Avant même que soit diagnostiqué le premier cas de CovID-19, le gouvernement de Hong Kong a mis en place plusieurs mesures. L'approche à volets multiples adoptée par Hong Kong (résumée dans l'encadré no 1) comprenait des contrôles frontaliers, des mesures d'éloignement sanitaire, la mise en quarantaine, la recherche active des contacts, des tests, du dépistage et des mesures de surveillance.

\section{Contrôles frontaliers et éloignement sanitaire}

Avant même le premier signalement sur son territoire, Hong Kong avait prévu qu'il y aurait des cas de COVID-19 et était sur un pied d'alerte. Le 3 janvier 2020, la vérification de la température à distance (par imagerie thermique) a été instaurée aux postes frontaliers pour les voyageurs qui arrivaient de Wuhan, en Chine, par train ou par avion ${ }^{2}$. Par la suite, les visiteurs en provenance de Wuhan et d'autres régions affectées de la Chine ont été refusés à

\section{POINTS CLÉS}

- Même si Hong Kong relie plusieurs routes internationales et se trouve près de Wuhan, en Chine, on y compte un nombre relativement faible de cas de maladie à coronavirus 2019 (COVID-19).

- Les mesures mises en place par les autorités de santé publique, comme les contrôles frontaliers, l'éloignement sanitaire, le volume élevé de tests de dépistage du coronavirus du syndrome respiratoire aigu sévère 2 (SRASCoV-2), la recherche active des contacts et la mise en quarantaine, ont probablement substantiellement contribué à maîtriser la situation, particulièrement pendant la période ayant suivi la confirmation du premier cas.

- L'adoption de mesures de protection personnelle, y compris le port du masque, par la plupart des résidents de Hong Kong peut avoir joué un rôle important dans le contrôle de la propagation du SRAS-CoV-2 dans la région.

la frontière de Hong Kong. Onze des 14 postes frontaliers de la ville ont été fermés sauf pour les déplacements essentiels, et les interventions de dépistage déployées auprès des voyageurs dans les aéroports et les postes frontaliers comprenaient la prise de la température corporelle et un questionnaire sur les symptômes de COVID-19. Les écoles primaires et secondaires ont été fermées par le ministère de l'Éducation le 27 janvier, suivies peu après des collèges et des universités. On a aussi demandé aux fonctionnaires de travailler à la maison (d'abord du 29 janvier au $1^{\text {er }}$ mars, puis à nouveau à partir du 23 mars; cette mesure est toujours en vigueur au moment d'écrire ces lignes). Le 25 mars, en raison du nombre croissant de cas chez les personnes de retour de l'étranger, le gouvernement de Hong Kong a interdit l'entrée à tous les nonrésidents en provenance de l'extérieur du territoire (à l'exception de Taiwan et de Macao) $)^{3}$. Le 29 mars, les rassemblements de plus de 4 personnes en public ont été interdits ${ }^{4}$, et le $1^{\text {er }}$ avril, les établissements de karaoké et de mah-jong, de même que les boîtes de nuit, ont dû fermer 5 .

\section{Recherche des contacts, dépistage et quarantaine obligatoire}

Le 19 mars 2020, une période de quarantaine de 14 jours a été rendue obligatoire pour quiconque arrivait à Hong Kong ${ }^{6}$. La recherche active des contacts et la mise en quarantaine des contacts 


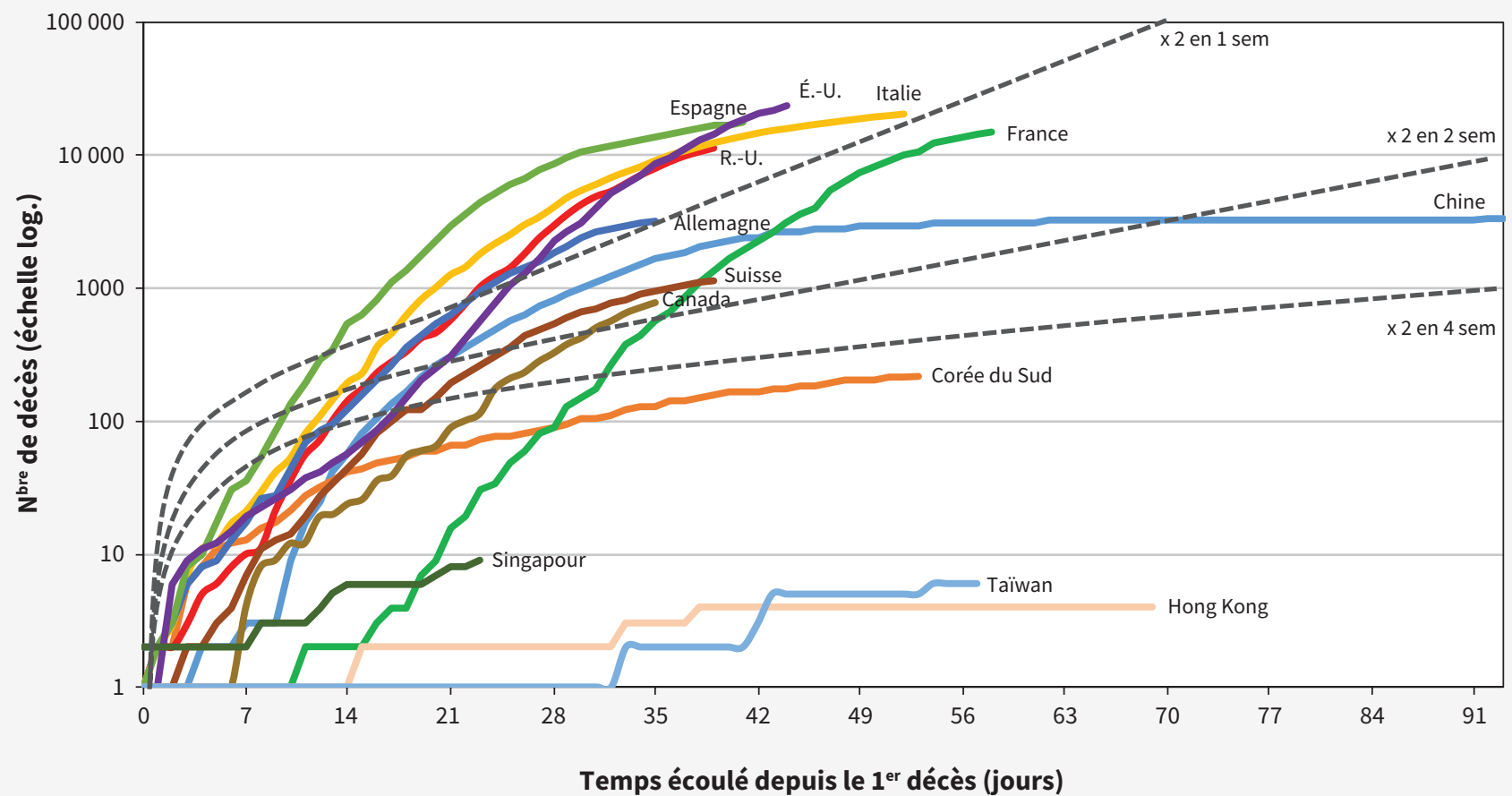

Figure 1 : Nombre cumulatif de décès attribuables à la COVID-19 (en date du 13 avril 2020). N.B. : L'unité de base des lignes de référence pour le doublement du nombre de cas est de 200 décès.

Encadré no 1: Mesures de santé publique appliquées par le gouvernement de Hong Kong durant la pandémie CoVID-19

Contrôles frontaliers et - Prise de la température aux postes frontaliers

éloignement sanitaire $\quad$ Questionnaire médical aux postes frontaliers

- Bannissement des voyageurs à haut risque (p. ex., d’abord les voyageurs provenant du Hubei, puis tous les nonrésidents de Hong Kong, et fermeture des frontières)

- Fermeture des écoles

- Télétravail

- Fermeture des établissements de loisirs et annulation des programmes récréatifs, sportifs et culturels

- Interdiction des réunions de plus de quatre personnes dans les endroits publics

- Fermeture des établissements de karaoké, de mah-jong et des boîtes de nuit (appliquée après la seconde vague de l'épidémie, en mars)

Recherche, quarantaine et

- Lieux de quarantaine désignés pour recevoir les contacts proches de cas confirmés et les personnes à haut risque arrivant à Hong Kong

dépistage des contacts

- Confinement obligatoire de certains contacts proches de cas confirmés et surveillance par bracelet électronique

- Tests à l'aéroport et à domicile des voyageurs de retour à Hong Kong; traitement ou hospitalisation des cas déclarés positifs organisé par les autorités sanitaires (www.chp.gov.hk/en)

Dépistage et surveillance

- Prélèvements de gorge chez les voyageurs arrivant à l'aéroport en provenance de pays comptant un nombre important de cas

- Programme de surveillance accrue par analyses de laboratoire des patients qui ont consulté dans une clinique de soins primaires ou un service d'urgence et de traumatologie alors qu'ils présentaient de la fièvre et de légers symptômes respiratoires

- Surveillance accrue par analyses de laboratoire dans les cliniques privées

Messages à la population

- Liste des établissements, des avions, des trains et des bateaux liés à des cas déclarés ainsi que des pays et des régions comptant des cas déclarés; conseils sanitaires et ligne téléphonique pour les cas soupçonnés fournis sur le site Web des autorités sanitaires

- Points de presse quotidiens

- Recommandations à la population de se protéger et de protéger les autres, notamment par le lavage des mains, le port du masque au besoin et le respect de l'étiquette respiratoire; on enjoint la population à sortir moins, à éviter les activités sociales et à respecter les recommandations en matière d'éloignement sanitaire 
proches des cas confirmés ont été appliquées, et plusieurs maisons de retraite, un terrain de camping et un nouveau développement domiciliaire ont été transformés en lieux de quarantaine. Les personnes asymptomatiques ayant été en contact étroit avec des cas infectés n'ont pas été testées pour la COVID-19. Par contre, ces personnes, de même que les voyageurs qui sont arrivés ou revenus à Hong Kong dans les 14 jours précédant le 25 mars après avoir séjourné dans une région comptant beaucoup de cas de COVID-19 (y compris Daegu et Gyeongsangbuk-do en Corée du Sud, l'Iran, ainsi que l'Émilie-Romagne, la Lombardie et la Vénétie en Italie), ont été envoyées dans des lieux de quarantaine. Ces lieux ne sont pas des établissements de santé, mais des sites d'hébergement où du personnel engagé par le ministère de la Santé de Hong Kong assure une surveillance et distribue des repas.

Les résidents de retour à Hong Kong après le 25 mars ont dû se soumettre à un confinement obligatoire de 14 jours sous surveillance par bracelet électronique et application mobile. Quiconque contrevenait à cette règle s'exposait à une condamnation en vertu du code criminel. En date du 30 mars, 54 personnes avaient été reconnues coupables de ce délit et envoyées dans des lieux de quarantaine ${ }^{7}$, et 3 personnes avaient été jugées coupables au sens de la loi et devaient purger des peines d'emprisonnement allant de 10 jours à 3 mois ${ }^{8}$.

\section{Dépistage et surveillance}

Afin de générer rapidement des données quant à la gravité de l'éclosion dans la communauté, on a mis en œuvre un programme intensif de surveillance par analyses de laboratoire des patients ayant consulté dans une clinique de soins primaires ou un service d'urgence et de traumatologie alors qu'ils présentaient de la fièvre et de légers symptômes respiratoires ${ }^{9}$. Ce programme, lancé le 13 janvier 2020 et toujours en vigueur, a fourni des données qui ont aidé les décideurs à déterminer si des cliniques de soins primaires désignées devaient être ouvertes pour les personnes présentant de la fièvre et des symptômes pertinents. Pour faciliter l'exactitude du dépistage, en milieu clinique, des cas de COVID-19 parmi les personnes chez qui l'infection était soupçonnée, le gouvernement a relié le système de dossiers du ministère de l'Immigration à celui des hôpitaux publics, fournissant ainsi un historique précis des déplacements.

Pour le dépistage, des tests diagnostiques ont été administrés à l'aéroport aux résidents de Hong Kong revenant de pays où la prévalence de la COVID-19 était élevée, comme les États-Unis, le Royaume-Uni, l'Italie et d'autres pays d'Europe. Pendant la deuxième vague de l'épidémie de COVID-19 à Hong Kong, au cours de laquelle la quasi-totalité des nouveaux cas signalés étaient des voyageurs ayant contracté l'infection à l'extérieur de Hong Kong, on a décrété un dépistage obligatoire du SRAS-CoV- $2^{10}$ au moyen de prélèvements de gorge réalisés dans une zone désignée de l'aéroport chez tous les arrivants qui avaient séjourné aux ÉtatsUnis, au Royaume-Uni, dans des pays d'Europe et dans la province de Hubei (indépendamment des symptômes), ainsi que chez ceux qui avaient 65 ans et plus ou qui résidaient avec des personnes de 65 ans et plus. Les personnes dont les résultats étaient positifs ont été hospitalisées pour être traitées, et celles qui avaient voyagé avec eux ont été envoyées dans des lieux de quarantaine. Les per- sonnes dont les résultats étaient négatifs pouvaient choisir de vivre leur quarantaine obligatoire de 14 jours à la maison ou dans un centre désigné (p. ex., un hôtel).

Ces mesures semblent avoir permis de maîtriser efficacement le risque d'une augmentation exponentielle du nombre de cas au début de l'épidémie ${ }^{11}$, et les mesures récentes semblent avoir ralenti l'augmentation des cas locaux (figure 2).

\section{Messages à la population}

Le site Web des autorités de santé publique de Hong Kong (Centre for Health Protection, www.chp.gov.hk/en) fournit une liste des établissements, des avions, des trains et des bateaux où ont circulé des passagers atteints de la COVID-19. Si une personne ayant obtenu un résultat positif au test de la COVID-19 s'est trouvée à bord d'un avion, le numéro du vol est inscrit sur le site Web. Les passagers qui se trouvaient à proximité de la personne infectée sont contactés, considérés comme des contacts proches et envoyés en quarantaine. Pour les autres passagers, l'information sur le site Web est fournie par souci de transparence, mais aussi pour qu'ils sachent qu'ils doivent surveiller l'apparition de symptômes (aucune autre action n'est entreprise par le gouvernement en ce qui concerne ces passagers). La liste des établissements fréquentés par des personnes infectées poursuit le même objectif de transparence dans le cadre de la stratégie de communication.

Des mises à jour sur le plus récent nombre de cas de COVID-19 dans les pays affectés, de même qu'une carte de Hong Kong illustrant les zones où des cas sont confirmés, sont accessibles sur ce site Web. On y fournit également des conseils santé ainsi que le numéro d'une ligne téléphonique pour les personnes soupçonnées d'avoir contracté la COVID-19, et le gouvernement et les autorités hospitalières présentent depuis janvier un point de presse quotidien sur la situation locale.

\section{Qu'a fait la population de Hong Kong pour réduire le risque d'infection?}

Forts de leur expérience avec l'épidémie de SRAS en 2002-2003, qui a entraîné 1755 infections et 299 décès à Hong Kong ${ }^{12}$, les résidents de la région ont adopté d'importantes mesures personnelles de protection contre la COVID-19. Dans un sondage mené auprès de la population générale quelques heures après la confirmation du premier cas d'infection, plus de $95 \%$ des répondants disaient se laver les mains fréquemment, et $99 \%$ disaient porter un masque ${ }^{13}$. Le port du masque est d'ailleurs devenu une norme culturelle à Hong Kong durant la présente pandémie.

Tous s'entendent pour dire que le lavage des mains est important pour maîtriser l'infection, mais le port du masque par les personnes asymptomatiques ne fait pas l'unanimité. À l'heure actuelle, l'Organisation mondiale de la santé ne recommande pas aux personnes asymptomatiques n'ayant pas eu de contacts étroits avec des personnes infectées de porter un masque chirurgical en public ${ }^{14}$. Cette recommandation tranche nettement avec les pratiques adoptées par les résidents des villes d'Asie orientale, comme Hong Kong, Taiwan et Singapour, où la plupart des gens ont choisi de porter un masque chirurgical dans les endroits publics ${ }^{15}$. 


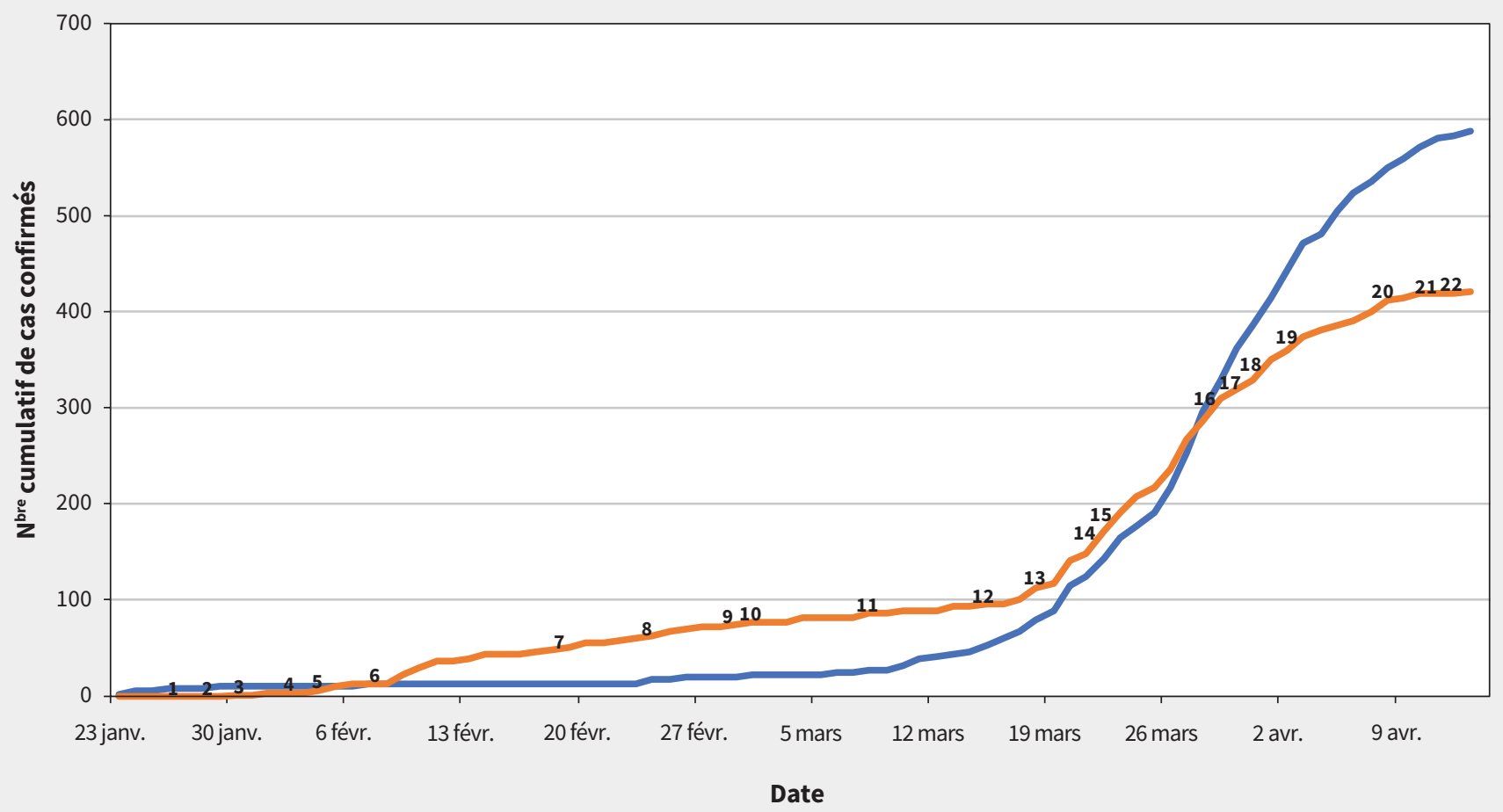

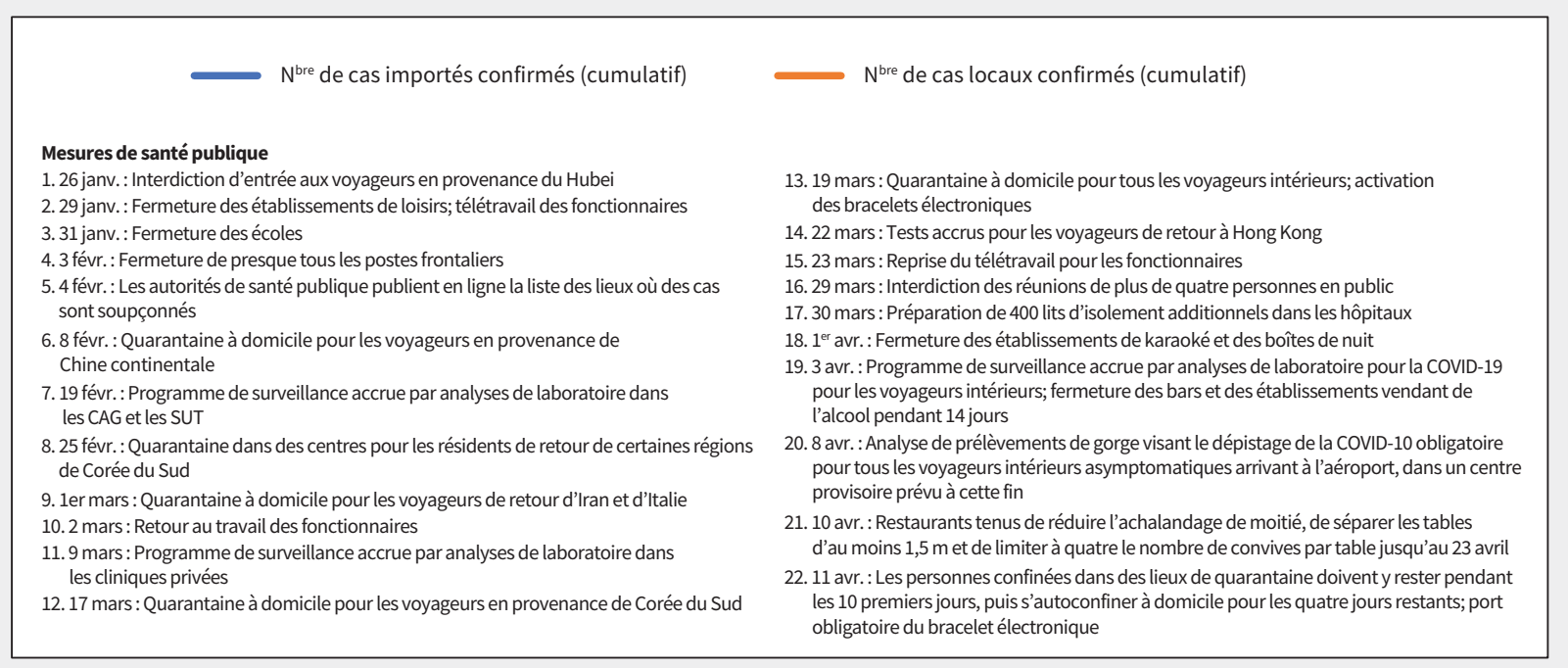

Figure 2 : Nombre de cas locaux et importés confirmés de COVID-19 à Hong Kong (du 23 janvier au 13 avril 2020), selon les autorités sanitaires de Hong Kong. CAG = clinique ambulatoire générale, SUT = service d'urgence et de traumatologie.

Pour l'instant, rien ne prouve que le port du masque en public aide à réduire le risque de maladies respiratoires, mais l'absence de données solides n'exclut pas la possibilité que cette pratique soit efficace. Il est difficile de procéder à des essais cliniques randomisés sur le sujet ${ }^{16}$. Selon une métaanalyse de $2015^{17}$, le port précoce du masque par des personnes en bonne santé pourrait protéger ces dernières contre la transmission de maladies grippales par des proches malades. De plus, des études cas-témoins réalisées durant l'éclosion de SRAS à Hong Kong ${ }^{18}$ et à Beijing ${ }^{19}$ ont indiqué, après neutralisation des facteurs de confusion potentiels comme l'âge, le sexe, les maladies chroniques, les visites dans un établissement de santé et la fréquentation de restaurants, que comparativement au port peu fréquent, le port régulier du masque (surtout du masque chirurgical) par la population générale dans les endroits publics pourrait avoir joué un rôle protecteur contre le SRAS. Combinées au port du masque, les pratiques de protection supplémentaires adoptées par la population générale durant l'épidémie de SRAS à Hong Kong ont substantiellement réduit l'incidence des infections virales respiratoires ${ }^{20}$.

Des cas de transmission du SRAS CoV-2 par des personnes asymptomatiques ont été signalés ${ }^{21}$, et on a établi que la charge virale peut être élevée durant la première phase de l'infection ${ }^{22}$. Un argument en faveur du port du masque par la population 
générale dans les endroits publics est que le masque peut potentiellement prévenir la transmission de la maladie par des porteurs asymptomatiques ${ }^{23}$.

Certains ont exprimé leur inquiétude que le port du masque chirurgical par la population générale entraîne une pénurie pour ceux qui en ont le plus besoin, comme les travailleurs de la santé24. On craint également qu'il donne aux gens un faux sentiment de sécurité ${ }^{14} \mathrm{ou}$, en cas d'utilisation inappropriée du masque, accroisse par inadvertance le risque d'infection ${ }^{25}$. Or, selon les résultats de notre sondage, le port du masque n'est pas associé à un taux moindre de lavage des mains ${ }^{13}$. De plus, on peut montrer aux gens à bien se servir d'un masque. Certains responsables de la santé publique ont récemment changé de position par rapport au masque, et des experts ont demandé l'adoption du principe de précaution et proposé le port universel du masque sur la base de certaines données en faveur de son efficacité ${ }^{11,23}$.

\section{Conclusion}

L'expérience que Hong Kong a acquise lors de l'épidémie de SRAS en 2003 pourrait expliquer pourquoi la santé publique et les résidents étaient si bien préparés et souhaitaient contrer rapidement la menace posée par la COVID-19. D'autres pays pourraient s'inspirer de l'approche active de confinement de Hong Kong, qui comprenait la recherche des contacts proches et leur mise en quarantaine obligatoire. Toutefois, chaque intervention de santé publique comporte des avantages et des inconvénients; une analyse minutieuse des facteurs contextuels locaux est nécessaire pour prendre les meilleures mesures possible.

\section{Références}

1. Latest situation of coronavirus disease (COVID-19) in Hong Kong. Hong Kong (China): Centre for Health Protection, Department of Health, The Government of the Hong Kong Special Administrative Region. Accessible ici : http:// chp-dashboard.geodata.gov.hk/covid-19/en.html (consulté le 14 avril 2020).

2. CE tours XRL health checkpoint. News.gov.hk 2020 Jan. 3. Accessible ici : www. news.gov.hk/eng/2020/01/20200103/20200103_193732_648.html?type= category\&name=wuhan\&tl=t (consulté le 18 mars 2020).

3. HK to ban non-resident arrivals. News.gov.hk 2020 Mar. 23. Accessible ici : www.news.gov.hk/eng/2020/03/20200323/20200323_164827_699.html (consulté le 2 avril 2020).

4. Group gathering ban gazetted. News.gov.hk 2020 Mar. 28. Accessible ici : www. news.gov.hk/eng/2020/03/20200328/20200328_110413_745.html (consulté le 2 avril 2020).

5. Karaokes, clubs, mahjong parlours ordered to close. Rthk.hk 2020 Apr. 1. Accessible ici : https://news.rthk.hk/rthk/en/component/k2/151821220200401.htm?spTabChangeable=0 (consulté le 2 avril 2020).

6. Government to impose mandatory quarantine on people entering Hong Kong from Mainland [press release]. Hong Kong (China): The Government of Hong Kong Special Administrative Region; 2020 Feb. 5. Accessible ici : www.info.gov. hk/gia/general/202002/05/P2020020500793.htm (consulté le 18 mars 2020).

7. 54 netted for quarantine breaches. News.gov.hk 2020 Mar. 26. Accessible ici : www.news.gov.hk/eng/2020/03/20200326/20200326_221347_495.html (consulté le 2 avril 2020)

8. 3 jailed for violating quarantine. News.gov.hk 2020 Mar. 30. Accessible ici : www.news.gov.hk/eng/2020/03/20200330/20200330_174317_389.html (consulté le 2 avril 2020).

9. DH sets up temporary specimen collection centre to speed up specimen collection from inbound travellers from overseas [press release]. Hong Kong (China): The Government of Hong Kong Special Administrative Region; 2020 Mar. 25. Available: www.info.gov.hk/gia/general/202003/25/P2020032500820. htm (consulté le 2 avril 2020).
10. DH strengthens health quarantine and testing arrangements for inbound travellers [press release]. Hong Kong (China): The Government of Hong Kong Special Administrative Region; 2020 Apr. 7. Accessible ici : www.info.gov.hk/ gia/general/202004/07/P2020040700753.htm (consulté le 14 avril 2020).

11. Cowling BJ, Ali ST, Ng TW, et al. Impact assessment of non-pharmaceutical interventions against COVID-19 and influenza in Hong Kong: an observational study. medRxiv 2020 Mar. 16. doi: 10.1101/2020.03.12.20034660.

12. Naylor CD, Chantler C, Griffiths S. Learning from SARS in Hong Kong and Toronto. JAMA 2004;291:2483-7.

13. Kwok KO, Li KK, Chan $\mathrm{HH}$, et al. Community responses during the early phase of the COVID-19 epidemic in Hong Kong: risk perception, information exposure and preventive measures. medRxiv 2020 Feb. 27. doi: 10.1101/2020.02.26.20028217.

14. Advice on the use of masks in the community, during home care and in healthcare settings in the context of the novel coronavirus (2019-nCoV)outbreak: interim guidance, 29 January 20202. Geneva: World, Health Organization; 2020. Accessible ici : https://apps.who.int/iris/handle/10665/330987 (consulté le 2 avril 2020).

15. Leung CC, Lam TH, Cheng KK. Mass masking in the COVID-19 epidemic: people need guidance. Lancet 2020;395:945.

16. Chan KH, Yuen KY. COVID-19 epidemic: disentangling the re-emerging controversy about medical facemasks from an epidemiological perspective. Int J Epidemiol 2020 Mar. 31 [Cyberpublication avant impression]. dyaa044. doi: 10.1093/ije/dyaa044.

17. MacIntyre CR, Chughtai AA. Facemasks for the prevention of infection in health care and community settings. BMJ 2015;350:h694.

18. Lau JT, Tsui H, Lau M, et al. SARS transmission, risk factors and prevention in Hong Kong. Emerg Infect Dis 2004;10:587-92.

19. Wu J, Xu F, Zhou W, et al. Risk factors for SARS among persons without known contact with SARS patients, Beijing, China. Emerg Infect Dis 2004;10:210-6.

20. Lo JY, Tsang TH, Leung YH, et al. Respiratory infections during the SARS outbreak, Hong Kong, 2003. Emerg Infect Dis 2005;11:1738-41.

21. Bai Y, Yao L, Wei T, et al. Presumed asymptomatic carrier transmission of COVID-19. JAMA 2020 Feb. 21 [Cyberpublication avant impression]. doi: 10.1001/jama.2020.2565.

22. Zou L, Ruan F, Huang M, et al. SARS-CoV-2 viral load in upper respiratory specimens of infected patients. N Engl J Med 2020;382:1177-9.

23. Greenhalgh T, Schmid MB, Czypionka T, et al. Face masks for the public during the covid-19 crisis. BMJ 2020;369:m1435.

24. Ranney ML, Griffeth V, Jha AK. Critical supply shortages - the need for ventilators and personal protective equipment during the COVID-19 pandemic. N Engl J Med 2020 Mar. 25 [Cyberpublication avant impression].doi: 10.1056/ NEJMp2006141.

25. Howard J. Masks may actually increase your coronavirus risk if worn improperly, surgeon general warns. CNN Health 2020 Mar. 2. Accessible ici : https://edition. cnn.com/2020/03/02/health/surgeon-general-coronavirus-masks-risk-trnd/index. html (consulté le 2 avril 2020).

Intérêts concurrents : Francis Chan a déclaré des honoraires personnels de Pfizer, d'AstraZeneca, d'Eisai, de Takeda Pharmaceuticals, d'EA Pharma et de la Société japonaise d'endoscopie gastroentérologique, et des subventions de Pfizer, d'AstraZeneca et de Takeda (Chine) pour la production de rapports. Aucun autre intérêt concurrent n'a été déclaré.

Cet article n'a pas été révisé par des pairs.

Affiliations : École de santé publique et de soins primaires du Jockey Club (Wong, Kwok) et Faculté de médecine (Chan), Université chinoise de Hong Kong, Hong Kong, Chine

Collaborateurs : Tous les auteurs ont contribué à l'élaboration et à la conception des travaux. Samuel Wong a rédigé la première version du manuscrit. Tous les auteurs ont révisé l'article, ont donné leur approbation finale pour la version destinée à être publiée et assument l'entière responsabilité de tous les aspects du travail.

Correspondance : Francis Chan, fklchan@cuhk.edu.hk 